\title{
TEMPERATURE DEPENDENCE OF PROPERTIES OF HETEROJUNCTIONS OF SOME TCNQ SALTS IN POLYMER MATRICES WITH $p$ - OR $n$-DOPED SILICON
}

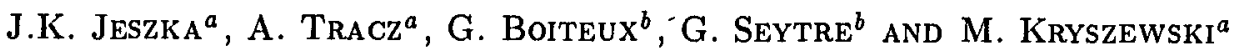 \\ a Centre of Molecular and Macromolecular Studies, Polish Academy of Sciences \\ Sienkiewicza 112, 90-363 Łódź, Poland \\ b LEMPB, Universite Lyon I, CNRS UA 507
}

43 Bd du 11 Novembre 1918, 69622 Villeurbanne, France

(Received April 19, 1995; in final form June 8, 1995)

\begin{abstract}
Dedicated to Professors Krzysztof Pigon, Józef W. Rohleder and Zdzisław Ruziewicz on the occasion of their 70th birthdays*
\end{abstract}

The temperature dependence of the electrical properties of heterojunctions with silicon formed by conductive organic polymer composites with networks of two complex tetracyanoquinodimethane salts (of $N$-n-butyl-isoquinolinium and of diethyl methyl sulphonium cations) were studied. We show that it is possible to prepare junctions with quite good rectifying properties, comparable to those obtained using other organic semiconductors. The observed forward-bias current-voltage characteristics can be satisfactorily fitted using the modified Schottky equation. Reverse bias and $C-V$ characteristics show that the transport mechanism, especially in the case of $p$-Si junctions is more complicated and probably tunnelling between localized levels plays an important role.

PACS numbers: 72.80.Le, 73.40.Lq

\section{Introduction}

It is well known that applications of inorganic semiconductors is mainly due to the electrical properties of their junctions which gave rise to numerous types of electronic devices of different characteristics. The properties of junctions of organic conductive polymers with metals or inorganic semiconductors like $\mathrm{Si}$

"Submitted on invitation of the Institute of Physical and Theoretical Chemistry, Technical University of Wrocław, Wrocław, Poland. 
or CdS have also been studied in recent years by several groups (see e.g. [1-4]), but they draw much less attention than bulk properties of organic conductors. Relatively few attempts were made to prepare and study the properties of junctions of low molecular weight organic semiconductors and they were practically limited to the compounds which can be deposited in a form of thin films by vacuum evaporation. The big group of charge-transfer (CT) complexes showing diverse interesting properties but obtained in a form of small crystals grown from solution have been considered not suitable for preparation of electronic devices.

Very recently polymer composite materials consisting of a fine network of low molecular weight organic CT complex crystallites penetrating inert polymer matrix have been used to prepare junctions [5-7]. Such materials, called reticulate doped polymers (RDP) can be obtained using a broad class of organic conductors and conventional insulating polymers by crystallization of the additive in situ [8-10]. It has been shown that networks of organic metals like well known tetrathiofulvalene-tetracyanoquinodimethane (TTF-TCNQ) or tetrathiotetracene-TCNQ (TTT-TCNQ) and complex TCNQ salts (in which $\mathrm{TCNQ}^{-}$and $\mathrm{TCNQ}^{0}$ coexist) can form rectifying junctions with silicon but junctions with metals were at most poorly rectifying $[5,6]$. It should be emphasized that the polymer matrix in RDP is inert and the electrical properties of such systems are determined by crystalline conductive networks i.e. by low molecular weight additives $[9,11]$.

In this paper we present the results of the studies of the temperature dependence of the electrical properties of junctions formed by RDP with networks of some complex TCNQ salts with silicon. The TCNQ salts which were selected are semiconductors and give fine, relatively well conductive networks in polymer films. Preliminary results at room temperature were presented in [7] and it was found that networks of both salts form rectifying junctions with both $n$-Si and $p$-Si. It is however well known that the properties of junctions formed by organic materials are often strongly temperature dependent and it is not possible to understand the transport mechanism without studies of the temperature dependence of junction parameters.

In the majority of papers on junctions of conductive polymer systems the temperature dependence of the device properties is not reported. Moreover, in many cases the barrier height is calculated assuming Richardson-Schottky mechanism and taking free-electron value of the Richardson constant (cf. e.g. [12, 13]), which is in strong disagreement with all published experimental results for organic semiconductors, which yield values by about 6 orders of magnitude lower. We show that also in the investigated systems such approach is not applicable and trap controlled tunnelling transport is probably the dominant mechanism.

\section{Experimental}

Conductive polymer films with fine networks of CT complex crystals were obtained by casting acetone solution containing proper amounts of the polymer and additives onto glass support or silicon wafer. Film thickness was about $20 \mu \mathrm{m}$. The vinylidene fluoride- $\mathrm{CH}_{2} \mathrm{~F}_{3} \mathrm{H}(6 / 4)$ copolymer obtained from Atochem (France) was used as polymer matrix and $N$-n-butyl-isoquinolinium (nBiQn) and diethyl methyl sulphonium $\left(\mathrm{SEt}_{2} \mathrm{Me}\right.$ ) complex TCNQ salts as conductive additives. Syn- 
thesis and electrical properties of $\mathrm{SEt}_{2} \mathrm{Me}(\mathrm{TCNQ})_{2}$ salt were described in [14] and $\mathrm{nBiQn}(\mathrm{TCNQ})_{2}$ was obtained from Sanyo (Japan). Both salts were purified by recrystallization. Interesting properties of $\mathrm{RDP}$ with $\mathrm{SEt}_{2} \mathrm{Me}(\mathrm{TCNQ})_{2}$ have been described in [10].

Heterojunctions with silicon were made using $n$ - or $p$-doped single crystal silicon wafers, $0.55 \mathrm{~mm}$ thick, $\langle 111\rangle$ crystal orientation and $3-10 \Omega \cdot \mathrm{cm}$ resistivity, doped with phosphorus or boron respectively. Wafers were etched in $48 \%$ aqueous IIF solution prior to junction preparation. Ohmic contacts to silicon were prepared by spark deposition of $\mathrm{Au}$ or $\mathrm{Au} / \mathrm{Sb}$ alloy on the back side. Their resistance was below $70 \Omega$, i.e. at least by an order of magnitude lower than series resistance of the investigated devices (see below). Junctions were prepared by casting RDP film on Si or by mechanical contact of the film (cast on glass support) with Si. The properties of devices carefully prepared using both methods were similar. All investigated devices were sandwich-type and the top electrode was $\mathrm{Au}\left(0.22 \mathrm{~cm}^{2}\right)$.

Electrical measurements were carried out in ambient atmosphere or in vacuum (the temperature dependences) and no significant differences in electrical properties were observed. Current-voltage $(I-V)$ characteristics were measured using Keithley 236 Source Measure Unit. Capacitance-voltage $(C-V)$ measurements were performed using Hewlett Packard 4284A LCR Meter in parallel R-C mode.

\section{Results and discussion}

Typical current-voltage $(I-V)$ characteristics of junctions formed by the polymer reticulate doped with 3 wt.\% of $n \mathrm{BiQn}(\mathrm{TCNQ})_{2}$ with $n-\mathrm{Si}$ and $p$-Si in semilogarithmic coordinates are presented in Fig. 1 (similar characteristics for $\mathrm{SEt}_{2} \mathrm{Me}(\mathrm{TCNQ})_{2}$ were presented in [7]). It can be seen that the junctions of the investigated RDP films with both $n$ - and $p$-doped silicon show quite good rectifying properties and the "forward" Si polarity is different for $n$-Si and $p$-Si.

The current-voltage characteristics of junctions of organic semiconductors showing rectifying properties can be analyzed using the ideal diode equation, modified to take into account also series resistance $R_{\mathrm{s}}$ of the device (including these of "ohmic" contacts), as well as imperfection of the barrier, represented by diode ideality factor $n$ and shunt (leakage) resistivity of the junction $R_{\mathrm{sh}}$

$$
J=J_{0}\left[\exp \frac{q\left(U-I R_{\mathrm{s}}\right)}{n k T}+1\right]+\frac{U-I R_{\mathrm{s}}}{R_{\mathrm{sh}}},
$$

$J$ denotes current density, $q$ - electron charge, $U$ - device voltage, $I$ - device current, $T$ - absolute temperature, $J_{0}$ - reverse saturation current and $k-$ Boltzmann constant. Junction voltage is therefore $V=U-I R_{\mathrm{s}}$. The current-voltage characteristics were fitted using computer program. Because the investigated RDP films measured in the sandwich configuration with two gold electrodes show superlinear $I-V$ characteristics with $I \propto U^{n}$, where $n$ was equal to about 1.4 for voltages above $0.2 \mathrm{~V}$, this effect was taken into account in the program as a suitable voltage dependence of $R_{\mathrm{s}}$. Similar voltage dependence of $R_{\mathrm{sh}}$ also improved the fits although it was generally not sufficient to obtain satisfactory fit of the reverse current $I_{\mathrm{R}}$. 


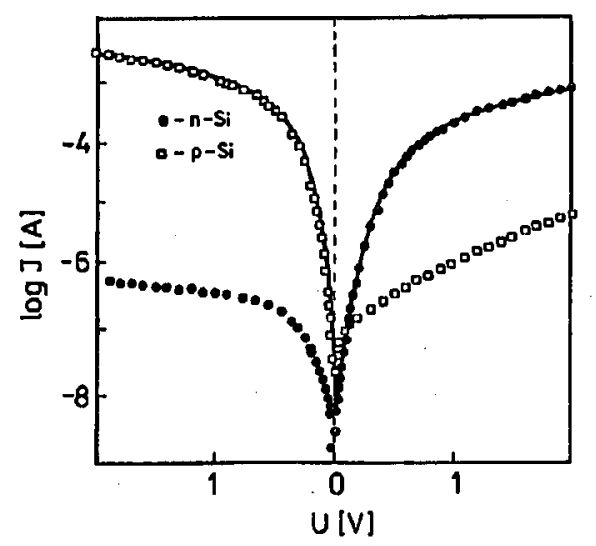

Fig. 1. $I-V$ characteristics of the polymer reticulate doped with nBiQn(TCNQ) 2 junctions with $n$-Si (o) and $p$-Si (प). Solid lines represent computer fits using the parameters listed in Table $\mathrm{I}$.

The values of $R_{\mathrm{s}}$ obtained from direct measurements on films provided with two metal electrodes in a sandwich configuration and from fittings were in reasonable agreement. The obtained resistivities were however about two orders of magnitude higher than measured by four-probe method in the perpendicular direction (in the plane of the film) which is due in part to conductive network anisotropy and in part to the metal contact resistivity.

Reverse bias characteristics are less reproducible than these for forward bias and we were unable to propose consistent interpretation of the obtained data. It is probably related to the complicated physical processes responsible for transport which give considerable contribution to the current at the reverse bias. For several samples at higher temperatures it was observed that at low voltage the current for reverse bias was higher than for forward bias.

The junction parameters obtained from computer fittings of the forward current $I_{F}$ are collected in Table I. One can see that the parameters differ depending on doping of Si and on the TCNQ salt forming the conductive network. The most important factors limiting rectification are series and shunt resistances. $R_{\mathrm{sh}}$ changes considerably depending on the conducting network morphology, sample geometry and junction preparation $\left(R_{\mathrm{s}}\right.$ does not change so much). Reproducibility of $n$ and $R_{\mathrm{s}}$ is of the order of $20 \%$ and of $J_{\mathrm{p}}$ about $50 \%$ for different samples.

In principle all device parameters in Eq. (1) are temperature dependent. The most important is however the temperature dependence of the reverse saturation current and of the ideality factor.

Temperature dependences of ideality factors $n$ and reverse saturation currents for investigated devices are presented in Figs. 2 and 3 respectively. It can be seen that in the case of $n \mathrm{BiQn}(\mathrm{TCNQ})_{2}$ junctions with both $n$-Si and $p$-Si the ideality factors are low and practically temperature independent while for $\mathrm{SEt}_{2} \mathrm{Me}(\mathrm{TCNQ})_{2}$ networks $n$ changes with temperature, especially for $n$-Si. In this system the values are the highest but still only slightly exceeding 2 , they are 
TABLE I

Junction parameters obtained by fitting the forward bias $I-V$ characteristics of the investigated junctions. R.t. denotes the value at room temperature. All activation energies are in $\mathrm{eV}$.

\begin{tabular}{c|c|c|c|c|c}
\hline \multicolumn{2}{c|}{ Junction parameters } & \multicolumn{2}{|c|}{$\mathrm{SEt}_{2} \mathrm{Me}(\mathrm{TCNQ})_{2}$} & \multicolumn{2}{c}{$\mathrm{nBiQn}(\mathrm{TCNQ})_{2}$} \\
\cline { 3 - 6 } \multicolumn{2}{l|}{} & $n-\mathrm{Si}$ & $p-\mathrm{Si}$ & $n-\mathrm{Si}$ & $p$-Si \\
\hline Rectification & r.t. & 11000 & 200 & 1400 & 800 \\
\hline$J_{0}\left[\mathrm{~A} / \mathrm{cm}^{2}\right]$ & r.t. & $3.5 \times 10^{-9}$ & $3.5 \times 10^{-7}$ & $1.2 \times 10^{-8}$ & $1.0 \times 10^{-7}$ \\
& $E_{\mathrm{a}}$ & 0.18 & 0.46 & 0.43 & 0.47 \\
\hline Ideality factor & r.t. & 2.0 & 1.6 & 1.9 & 1.2 \\
\hline$R_{\mathrm{s}}[\mathrm{k} \Omega]$ & r.t. & 0.9 & 0.7 & 2.5 & 0.6 \\
& $E_{\mathrm{a}}$ & 0.043 & 0.045 & 0.010 & 0.043 \\
\hline$R_{\mathrm{sh}}[\mathrm{M} \Omega]$ & r.t. & 15 & 0.35 & 4 & 1 \\
& $E_{\mathrm{a}}$ & 0.21 & 0.45 & 0.31 & 0.37
\end{tabular}

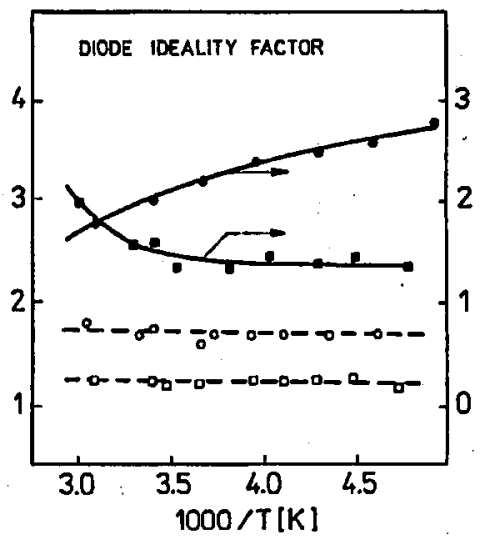

Fig. 2. Temperature dependences of the diode ideality factors for the investigated junctions. Empty symbols correspond to $n \operatorname{BiQn}(\mathrm{TCNQ})_{2}$ and full symbols to $\mathrm{SEt}_{2} \mathrm{Me}(\mathrm{TCNQ})_{2}(\mathrm{o} n-\mathrm{Si}$ and $\square p-\mathrm{Si})$.

quite good as compared with other organic semiconductors $[1,3]$. Non-ideality of the investigated devices is most probably related to the surface states of the RDP film and their position relative to the Fermi level determines their importance in the given device. Reverse saturation currents show similar temperature dependences except for $n$-Si/SEt ${ }_{2} \mathrm{Me}(\mathrm{TCNQ})_{2}$ junctions which is related to $n$ variation for these devices.

Capacitance-voltage $(C-V)$ measurements also show complicated and poorly reproducible dependences, related to the phenomena controlling reverse bias currents $(C-V$. characteristics can be measured for reverse bias and small forward voltages). The second reason of these difficulties is that junction capacitance is 


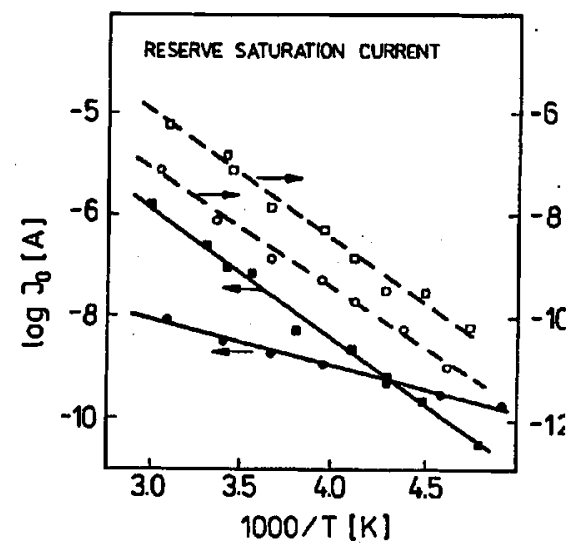

Fig. 3. Temperature dependences of the reverse saturation currents for the investigated junctions. Empty symbols correspond to $\mathrm{nBiQn}(\mathrm{TCNQ})_{2}$ and full symbols to $\mathrm{SEt}_{2} \mathrm{Me}(\mathrm{TCNQ})_{2}(\circ n-\mathrm{Si}$ and $\square p$-Si).

not measured directly but must be calculated from the equivalent circuit including $R_{\mathrm{s}}, R_{\mathrm{sh}}$ and the capacitances of the sample and metal/polymer contact $[1,4]$. In principle it is possible to evaluate these values from independent measurements but their poor reproducibility and anomalous behaviour around zero bias made these calculations generally not satisfactorily reliable. In spite of the corrections for the capacitance of the conductive network and the resistances $R_{\mathrm{s}}$ and $R_{\mathrm{rs}}$ (even taking into account their voltage and frequency dependences measured in independent experiments) the plots of $1 / C^{2}$ vs. junction voltage are generally not linear, especially around 0 bias (c.f. Fig. 2 in [7]). It suggests that the charge transport in the investigated devices is more complicated. Most probably the other parallel transport mechanism through the junction is hopping between localized levels. The data obtained for $n$-Si were presented in [9] and the extrapolations yield the barrier heights similar to $I-V$ characteristics in this case (Table II). We were unable to obtain for $p$-Si the data which could be interpreted in this way.

Beside the junction built-in voltage (which can be obtained from fitting using Eq. (1)) and capacitance-voltage characteristics discussed above, the temperature dependence of the reverse saturation current can be used to determine the barrier height on the rectifying junction.

In general, several mechanisms contribute to the reverse saturation current. The most important are: emission over the top of the barrier (described by thermionic emission theory), transport through the depletion region (diffusion theory) and indirect tunnelling through the barrier (tunnel recombination mechanism) [1]. Assuming that one of the first two dominates the current, $J_{0}$ can be written as

$$
J_{0}=J^{*} \exp (q \Phi / k T)+J_{\mathrm{t}} \exp \left(E_{\mathrm{t}} / k T\right)
$$

where $\Phi-$ barrier height, $E_{\mathrm{t}}-$ trap depth, $J_{\mathrm{t}}-$ tunnelling saturation current (proportional to the density of traps participating in this mechanism) [1].

In the case of Richardson-Schottky mechanism $J^{*}=A^{*} T^{2}$, where $A^{*}$ is the so-called Richardson constant and in the case of organic semiconductors it is 
TABLE II

Junction parameters determined using different methods (see text for details).

\begin{tabular}{l|c|c|c|c}
\hline \multirow{2}{*}{ Barrier parameters } & \multicolumn{2}{|c|}{$\mathrm{SEt}_{2} \mathrm{Me}(\mathrm{TCNQ})_{2}$} & \multicolumn{2}{c}{$\mathrm{nBiQn}(\mathrm{TCNQ})_{2}$} \\
\cline { 2 - 5 } & $n-\mathrm{Si}$ & $p-\mathrm{Si}$ & $n-\mathrm{Si}$ & $p$-Si \\
\hline Built-in voltage & & & & \\
$(I-V$ characteristics $)$ & $0.6-0.7$ & $0.35-0.5$ & $0.5-0.65$ & $0.34-0.4$ \\
$(C-V$ characteristics) & 0.66 & - & 0.52 & - \\
\hline From saturation current & & & & \\
Richardson constant & $10^{-4}$ & $1.4 \times 10^{-4}$ & $2.6 \times 10^{-7}$ & $2.5 \times 10^{-5}$ \\
Diffusion constant & 3 & 1.9 & 0.15 & 12 \\
$E_{\mathrm{a}}$ (from log $J_{0}$ vs. $\left.1 / T\right)$ & 0.18 & 0.46 & 0.43 & 0.47 \\
\hline$N_{\mathrm{a}}$ (in additive) & $1 \times 10^{18}$ & - & $7 \times 10^{17}$ & - \\
Barrier width $W$ & $65 \mathrm{~nm}$ & - & $70 \mathrm{~nm}$ & - \\
Mobility $\mu\left[\mathrm{cm}^{2} /(\mathrm{V} \mathrm{s})\right]$ & $3 \times 10^{-3}$ & - & $4 \times 10^{-3}$ & -
\end{tabular}

usually assumed to be equal to the free electron value $120 \mathrm{~A} /\left(\mathrm{cm}^{2} \mathrm{~K}^{2}\right)$ (the only material-dependent variable that enters into $A^{*}$ is the charge carrier effective mass so it should not be much different from this value in organic materials).

In the case of diffusion mechanism $J^{*}=q N_{\mathrm{s}} \mu E$. Here $N_{\mathrm{s}}$ denotes effective density of states, $\mu$ - charge carrier mobility and $E$ - maximum electric field in the junction. This mechanism seems to be much more appropriate in most organic systems.

The temperature dependences of $J_{0}$ for the investigated junctions of RDP film plotted in the coordinates $J_{0} / T^{2}$ vs. $1 / T$ (Richardson plot) yield the values of the Richardson constant $10^{-4}-10^{-6} \mathrm{~A} /\left(\mathrm{cm}^{2} \mathrm{~K}^{2}\right)$ (see Table II) instead of $120 \mathrm{~A} /\left(\mathrm{cm}^{2} \mathrm{~K}^{2}\right)$. The diffusion mechanism seems therefore much more likely to operate in the investigated devices. The plots of $J_{0}$ vs. $1 / T$ presented in Fig. 3 are approximately linear, which allows us to determine activation energies.

Junction parameters determined using different methods or models are compared in Table II. The build-in voltage was estimated from the saturation value of the junction voltage (calculated by subtracting the voltage drop on $R_{\mathrm{s}}$ from the voltage applied) and from $C-V$ characteristics. It can be seen that for $n$-Si the voltage values determined from $C-V$ and from $I-V$ characteristics at room temperature are in good agreement. The activation energies obtained from the temperature dependence are of the same order as the built-in voltages or smaller, especially for $\mathrm{SEt}_{2} \mathrm{Me}(\mathrm{TCNQ})_{2}$ for which the ideality factor is strongly temperature dependent. In many cases it has been found that the effect of the temperature variation of these two parameters is opposite so some "compensation effect" is observed $[1,4,6]$. In the investigated systems this effect was observed for $n$-Si contacts but not for $p$-Si as it can be seen in Fig. 3.

In the case of $p$-Si the data from $I-V$ characteristics agree reasonably with 
the temperature dependence but $C-V$ dependences were not linear so this method could not be used.

Assuming the simplified (but commonly used for organic materials) model of the junction we can calculate from $C-V$ characteristics the ionized donor concentrations and barrier widths $[1,15,16]$ which are also listed in Table II. Consequently expressing conductivity as $\sigma=q \mu N_{\mathrm{a}}$ we can also calculate charge carrier mobilities $\mu$. It should be emphasized that these values calculated using this oversimplified model should be treated as order-of-magnitude estimations only. The values of $N_{\mathrm{s}}$ and $\mu$ are however in reasonable agreement with those found for similar systems [4].

However, the maximum electric field $E$ calculated from the diffusion constant and conductivity seems to be about an order of magnitude too high as compared with that calculated by dividing junction voltage by barrier width.

Some inconsistency appears also in the barrier heights calculations. In principle it can be expressed as $\Phi=V_{\mathrm{bi}}-\left(E_{\mathrm{F}}-E_{\mathrm{v}}\right)$ where $E_{\mathrm{F}}$ and $E_{\mathrm{v}}$ are Fermi energy and valence band energies respectively. The difference of these energies should be at least of the order of magnitude of the activation energy of conductivity, i.e. about $0.04 \mathrm{eV}$, while from Table II it can be seen that activation energy of the reverse saturation current (which for both Richardson and diffusion mechanism should be equal to barrier height $\Phi$ ) is lower than the build-in voltage. It suggests that an important contribution to the reverse saturation current comes from the tunnelling mechanism in the charge carrier transport through the investigated junctions (the second term in Eq. (2)) and therefore $E_{\mathrm{a}}$ should be interpreted rather as the trap depth $E_{\mathrm{t}}$.

\section{Conclusions}

Using conductive polymer composites with crystalline networks of complex TCNQ salts we prepared rectifying junctions with both $n$-type and $p$-type silicon and studied the temperature dependence of their electrical characteristics. $I-V$ characteristics for the forward bias can be fitted using modified Schottky equation but to explain the reverse bias and $C-V$ characteristics additional effects must be taken into account.

The temperature dependences of the reverse saturation currents show that Richardson-Schottky mechanism cannot be used to determine the barrier heights in the investigated devices and diffusion and (especially for reverse bias) indirect tunnelling through the barrier (tunnel recombination) mechanisms give the most important contributions to the current.

Comparing with previously used conductive polymers or networks of molecular metals, polymers reticulate doped with the investigated additives give junctions with silicon showing similar or better properties.

\section{References}

[1] J. Kanicki, in: The Handbook of Conducting Polymers, Ed. T.A. Skotheim, Vol. 2, Marcel Dekker, New York 1986, p. 543.

[2] M.J. Sailor, F.L. Klavetter, R.H. Grubbs, N.S. Levis, Nature 346, 155 (1990). 
[3] J.S. Glenis, A.J. Frank, Synth. Met. 28, C681 (1989).

[4] J.K. Jeszka, Mater. Sci. Forum, 1995, in press.

[5] B. Remaki, D. Jullien, C. Jouve, Sens. Actuators A 33, 85 (1992).

[6] J.K. Jeszka, A. Tracz, M. Kryszewski, Synth. Met. 64, 203 (1994).

[7] J.K. Jeszka, A. Tracz, G. Boiteux, G. Seytre, M. Kryszewski, Synth. Met. 71, 2205 (1995).

[8] M. Kryszewski, J. Ulański, J.K. Jeszka, A. Tracz, Pure Appl. Chem. 56, 355 (1984).

[9] M. Kryszewski, J. Ulański, in: The Encyclopedia of Advanced Materials, Eds.

D. Bloor, M.C. Flemings, R.J. Brook, S. Mahajan, Pergamon Press, London 1993, p. 2301.

[10] J. Pęcherz, L. Firlej, J.K. Jeszka, J. Ulański, M. Kryszewski, Synth. Met. 37, 189 (1990).

[11] J. Ulaniski, Synth. Met. 39, 13 (1990).

[12] A. Watanabe, S. Murakami, K. Mori, Y. Kashiwaba, Macromolecules 22, 4231 (1989).

[13] C.S. Kuo, F.G. Wakim, S.K. Sengupta, S.K. Tripathy, J. Appl. Phys. 74, 2957 (1993).

[14] J. Pęcherz, M. Kryszewski, L. Firlej, A. Graja, Synth. Met. 35, 39 (1990).

[15] S.M. Sze, Physics of Semiconductor Devices, Wiley, New York 1989.

[16]'E.H. Rhoderick, Metal-Semiconductor Contacts, Calderon Press, Oxford 1980. 